differentiation between wind and solid had a positive correlation with better QOL ( $p=0.007$ and 0.02 respectively). There was a nonsignificant decrease in the overall $\mathrm{QOL}$ (EQ-5D $\mathrm{p}=0.6$, EQ-VAS $\mathrm{p}=0.12$ ) following hemicolectomy compared with controls.

Conclusion Colonic resection has an adverse effect on bowel frequency with worse outcomes following right hemicolectomy. Good colonic function, as assessed by the MSKCC questionnaire, is associated with better QOL.

Disclosure of Interest None Declared

\section{PWE-002 SHORT-TERM OUTCOMES FOLLOWING SURGICAL TREATMENT OF BENIGN COLONIC POLYPS: A CASE- MATCHED STUDY}

doi:10.1136/gutjnl-2013-304907.291

1,"A Brigic, ${ }^{2} \mathrm{R}$ Cahill, 'G J Williams, ' ${ }^{\mathrm{H}} \mathrm{A}$ Alexander, ' $\mathrm{J}$ T Jenkins, ${ }^{3} \mathrm{C}$ Fraser, 'S K Clark, 'R H Kennedy. 'Department of Surgery, St. Mark's Hospital, London, UK; ${ }^{2}$ Department of Surgery, Beaumont Hospital, Dublin, Ireland; 'Wolfson Endoscopy Unit, St. Mark's Hospital, London, UK

Introduction Patients diagnosed with complex colonic polyps (broad based, crossing two haustral folds, or being located at the ileocaecal valve or colonic flexures) unsuitable for endoscopic treatment undergo the same surgical procedure as patients with colon cancer. As a result of the bowel cancer screening programmes, the number of these patients has increased significantly and outcomes after hemicolectomy for benign colonic polyps (BCP) are poorly documented. We present a case-matched study examining shortterm outcomes of patients with BCP versus those with colonic cancer (CC) from two institutions.

Methods Consecutive patients undergoing surgery for BCP were identified in two hospitals from prospectively maintained databases (data collection period 2005-2006 and 2010-2012 respectively). Hospital coding database was also searched using operation codes to identify missing patients. Each patient was matched for age, sex, ASA grade, site and type of resection (laparoscopic, open, and converted) to two controls undergoing surgery for treatment of CC identified in each centre. The length of stay (LOS) and 30-day outcomes were analysed with further adjustments for BMI, blood loss and operation time. Multilevel linear and logistic regression analyses were performed.

Results 46 BCP patients were matched with 81 CC patients. Almost all procedures were performed laparoscopically (42/46). Two procedures were converted to open and two patients underwent planned open surgery. Median size of BCP was $4 \mathrm{~cm}$ (IOR 2.5, 5.4). BCP group had a marginally longer LOS [median 5.5 days (IOR 4, 8) and 5 days (IOR 3,7$)$ respectively $(p=0.04)]$. 21/46 $(46 \%)$ patients with BCP had a postoperative complication compared to $25 / 81$ (31\%) CC patients $(p=0.12, \mathrm{OR}=2.11$; CI 0.82-5.41). 4/46 $(9 \%)$ BCP patients underwent reoperation and further $3 / 46(7 \%)$ were readmitted versus $1 / 81(1 \%)$ and $2 / 81(2 \%)$ in CC group ( $p=0.07$ and 0.28 respectively). No deaths were observed in either group.

Conclusion Complications following segmental colectomy for complex colonic polyps are not significantly different to those after cancer surgery. The results of this study provide further impetus for the development of a local full thickness colonic excision technique as an alternative, less invasive treatment option in order to improve patient outcomes.

Disclosure of Interest None Declared

\section{PWE-003 COLORECTAL CANCERS ARISING IN ADENOMAS ("POLYP CANCERS") DISCOVERED AND RESECTED DURING BOWEL CANCER SCREENING COLONOSCOPY}

doi:10.1136/gutjnl-2013-304907.292

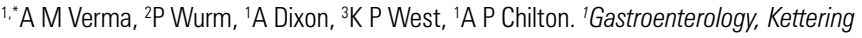
General Hospital NHS foundation trust, Kettering; ' ${ }^{2}$ Gastroenterology; ${ }^{3}$ Pathology, University Hospitals Leicester NHS trust, Leicester, UK
Introduction The national bowel cancer screening programme (BCSP) was rolled out in 2006. Screening is based on a guaiac FOBt strategy, positive tests are offered colonoscopy. In January 2008 implementation commenced in LNR region (pop $\sim 1.6$ million).

Polyp cancers present a challenge both to clinicians and patients - optimal management remains unclear. We prospectively followed all patients who underwent primary endoscopic polypectomy.

Methods Patients with polyp cancers resected by polypectomy alone within the BCSP (48 cases as of 31/12/11) have had their outcomes recorded (last update 31/10/12).

Results Demographic data

Mean $/$ median age $=65.92 / 66 \mathrm{yrs}$

Males/Females $=33(68.75 \%) / 15(31.25 \%)$

Location of polyp cancer

Rectum $=5(10.4 \%) /$ Sigmoid $=41(85.4 \%) /$ Desc colon $=2(4.2 \%)$

\section{Abstract PWE-003 Table}

\begin{tabular}{|c|c|c|c|c|c|}
\hline & \multicolumn{4}{|c|}{ Pedunculated polyp cancers - 35 cases } & \multirow{2}{*}{$\begin{array}{l}\text { Other } \\
\text { polyp } \\
\text { cancers }\end{array}$} \\
\hline & Haggitt 1 & Haggitt 2 & Haggitt 1 \& 2 & Haggitt 3 & \\
\hline Number & 15 & 11 & 26 & 9 & 13 \\
\hline Recurrences & 0 & 0 & 0 & 0 & 1 \\
\hline $\begin{array}{l}\text { Mean time post index } \\
\text { polypectomy }\end{array}$ & $\begin{array}{l}\text { x33.7 months } \\
(11.5-50.1)\end{array}$ & $\begin{array}{l}34.1 \text { mths } \\
(16.4-52.2)\end{array}$ & $\begin{array}{l}33.8 \text { mths } \\
(11.5-52.2)\end{array}$ & $\begin{array}{l}40.4 \text { mths } \\
(27.0-54.5)\end{array}$ & $\begin{array}{l}\text { see } \\
\text { notes }\end{array}$ \\
\hline $\begin{array}{l}\text { Cumulative } \\
\text { recurrence free time }\end{array}$ & $\begin{array}{l}504.8 \text { months } \\
\text { (42.1 yrs) }\end{array}$ & $\begin{array}{l}375 \mathrm{mths} \\
(31.2 \mathrm{yrs})\end{array}$ & $\begin{array}{l}879.8 \mathrm{mths} \\
\text { (73.3 yrs) }\end{array}$ & $\begin{array}{l}363.3 \mathrm{mths} \\
(30.3 \mathrm{yrs})\end{array}$ & below \\
\hline
\end{tabular}

Notes One patient with a Haggitt 1 polyp cancer has subsequently died from ascending colon adenocarcinoma (a new lesion). Of the 13 other polyp cancers, 5 were pedunculated but not assigned Haggitt levels due to incomplete excision or invasive cancer - one of these patients has subsequently died with liver metastases. There were 8 sessile polyp cancers with no recurrences - too small a group to draw any meaningful conclusions.

Conclusion There were 26 patients with Haggitt 1 or 2 polyp cancers. Cumulative follow up of 73.3 yrs has not identified any case of recurrence, suggesting that endoscopic resection of these lesions is curative. The 9 patients with Haggitt 3 polyp cancers (invasion into stalk) have 30.3 yrs cumulative recurrence free time.

It is recognised that there is an increased risk of adverse outcomes (lymph node metastasis/recurrence $)^{1}$ from Haggitt 4 polyp cancers (invasion into bowel wall submucosa below stalk) and hence factors such as adequate resection margins (despite diathermy artefact) and length of stalk have to be taken into account to ensure that Haggitt 3 polyp cancers are not actually Haggitt 4 polyp cancers.

Patients with Haggitt 3 polyp cancers, where there is evidence of adequate resection margins, should be offered the option of conservative treatment balanced with the risks of formal resection.

Disclosure of Interest None Declared

\section{REFERENCE}

1. Haggitt RC, Glotzbach RE, Soffer EE, Wruble LD. Prognostic factors in colorectal carcinomas arising in adenomas: implications for lesions removed by endoscopic polypectomy. Gastroenterology 1985; 89:328-36.

\section{PWE-004 DETECTION OF VENOUS INVASION TO STAGE COLORECTAL CANCER}

doi:10.1136/gutjnl-2013-304907.293

1.*A K Foulis, C S D Roxburgh, D C McMillan, C H Richards, M Atwan, J H Anderson, T Harvey, P G Horgan. 'Department of Pathology, Southern General Hospital, Glasgow, UK

Introduction Elastica staining of tumour sections increases the sensitivity of detection of venous invasion in colorectal cancer. We compared the prognostic value of elastica detected venous invasion with that of other pathological features in colorectal tumours. 
Methods This was a single-centre clinical outcome study of pathological variables, including elastica detected venous invasion, in stage I-III electively resected colorectal cancer specimens.

Results 631 resection specimens, excised between 1997-2009, were analysed (176 retrospectivley and 455 prospectively). The median follow up was 73 months (24-178) and during this time there were 238 deaths (134 from cancer). Venous invasion was detected in $56 \%$ of cases and was a stronger predictor of poor long term cancer-specific survival than other pathological features on univariate and multivariate analyses. On multivariate analysis of all cases the hazard ratio (HR) for failure to survive 5 years for venous invasion $=3.94$ (95\%CI 2.33-6.65, P < 0.001); HR for lymph node involvement $=1.81,(95 \%$ CI 1.43-2.30, $\mathrm{P}<0.001)$ and $\mathrm{HR}$ for $\mathrm{T}$ stage $=1.64(95 \% \mathrm{CI} 1.16-2.30, \mathrm{P}=0.005)$. In node negative cases the HR for failure to survive 5 years for venous invasion on multivariate analysis $=3.55(95 \% \mathrm{CI} 1.81-6.97, \mathrm{P}<0.001)$ and for $\mathrm{T}$ stage was $2.03(95 \%$ CI $1.26-3.28, \mathrm{P}=0.004)$. Venous invasion strongly related to other high-risk pathological variables. In cases with no venous invasion, no pathological characteristic related to survival other than $\mathrm{T}$ stage. When $\mathrm{T}$ stage and venous invasion were considered together, patients could be stratified by risk of 5 -year cancer mortality from $100-54 \%$ in node negative disease and $100-33 \%$ in node positive disease.

The importance of elastica detected venous invasion can be appreciated from the development of a novel staging system based only on T stage and venous invasion (TVI). This simple TVI system was at least as predictive as the gold standard TNM system when considering all cases, and provided increased prognostic value in both T1 and T2 tumours, as well as in node negative disease.

Conclusion Sensitive, accurate detection of venous invasion on elastica stained sections improves its prognostic importance such that it becomes a key pathological characteristic, arguably of more importance than nodal status, in determining outcome in patients with colorectal cancer. TVI staging provides a novel and simple method by which venous invasion coupled with T stage can be utilised to predict survival.

Disclosure of Interest None Declared

\section{PWE-005 IS INVESTIGATION OF THE COLONIC LUMEN NECESSARY AFTER CT DIAGNOSIS OF ACUTE DIVERTICULITIS?}

doi:10.1136/gutjnl-2013-304907.294

1."A Tan, ' $\mathrm{C}$ Newman. ' Colorectal Surgery, Airedale NHS Foundation Trust, Keighley, UK

Introduction Traditionally, it is recommended that the colon be evaluated after an episode of acute diverticulitis to exclude underlying pathologies such as malignancy. However, the necessity of this has been questioned in this era of frequent use of contrast-enhanced multidetector computed tomography (CT), which itself is a moderately sensitive test for colorectal cancer, to diagnose acute diverticulitis. This study aimed to determine the yield of malignancy from subsequent follow-up investigations or treatments in patients after an episode of CT-proven acute diverticulitis.

Methods This was a retrospective study of patients with acute diverticulitis as diagnosed on CT at a moderately-sized district general hospital between January 2007 and December 2011. Uncomplicated diverticulitis was defined as the presence of colonic diverticular disease with localised colonic wall thickening and/or inflammation of pericolic fat. Complicated diverticulitis was defined as the additional presence of abscess, obstruction, fistula or evidence of perforation.

Results A total of 64 patients were diagnosed with acute diverticulitis on contrast-enhanced CT. Thirty-five patients had uncomplicated diverticulitis and 29 patients had complicated diverticulitis.

The follow-up of patients with uncomplicated disease were as follows: flexible sigmoidoscopy (10), colonoscopy (4), CT colonography
(2) and barium enema (4). Two patients underwent surgery. All investigations or treatments confirmed diverticular disease. None of them showed underlying malignancy. Incidental findings at the diseased sites were detected in 4 patients. Three of them had polyps and one had an aphthous ulcer. Of the patients with polyps, one had a tubular adenoma greater than $10 \mathrm{~mm}$, one had a $2 \mathrm{~mm}$ tubular adenoma with low-grade dysplasia and one had an inflammatory polyp. The biopsy of the patient with the ulcer was normal. The remaining 13 patients had no follow-up and none of them were known to have returned with malignancy.

The follow-up of patients with complicated disease were as follows: flexible sigmoidoscopy (9), colonoscopy (4), CT colonography (1) and barium enema (5). Six patients had surgery. All investigations or treatments confirmed diverticular disease with no evidence of underlying malignancy. A patient who underwent surgery incidentally had hyperplastic polyps in the sigmoid colon specimen. Four patients had no follow-up and none of them were known to have developed malignancy.

Conclusion The yield of malignancy as an underlying pathology was nil in both cohorts. Routine investigation of the colonic lumen after an episode of acute diverticulitis in this era of widespread CT use may not be necessary in the absence of other sinister indications. Limited resources may be better utilised for other patients with greater risk of colorectal cancer.

Disclosure of Interest None Declared

\section{PWE-006 HOW OFTEN IS BOWEL CANCER DETECTED FROM A POSITIVE 3RD KIT IN THE ENGLISH BCSP?}

doi:10.1136/gutjnl-2013-304907.295

1."A Field, 'M Vogler, ${ }^{1} \mathrm{R}$ F Logan. 'Eastern Bowel Cancer Screening Hub, Nottingham University Hospitals Trust, Nottingham, UK

Introduction In the English Bowel Cancer Screening Programme (BCSP) subjects returning a weak positive kit (1-4 of the 6 windows positive) are invited to do a $2^{\text {nd }}$ kit and if none of the windows are positive they are invited to complete a $3^{\text {rd }}$ kit. If any windows are then positive subjects are referred for possible investigation; if no windows are positive subjects are discharged from that screening round. This testing algorithm has been criticised for making the screening process too prolonged thereby producing anxiety and drop-outs and the Scottish BCSP has abandoned asking for a $3^{\text {rd }}$ kit on the grounds that the yield was negligible.

Methods We have analysed the outcomes from the $3^{\text {rd }}$ kits returned to the Eastern BCSP Hub from subjects invited for screening between 1 Jan 2011 and 31 March 2012.

Results Over this period over 850,000 subjects aged 60-74 yrs were invited for screening. $4 \%(20,021)$ completed 3 kits and of these $16 \%$ (3192) had a positive $3^{\text {rd }}$ kit and were referred for further investigation. Of those investigated (2830) 4.4\% (125) were found to have bowel cancer compared with $17.8 \%$ (298) with cancer found after a single kit and $8.1 \%$ (483) with cancer found after completing 2 kits. A further $7 \%$ and $12 \%$ completing 3 kits were found to have high and intermediate risk adenomas. The mean time from selection for screening to obtaining a definitive result for those completing 3 kits was 63 days compared to 34 days for those completing a single kit ( $95 \%$ of all subjects returning kits) and 49 days for those completing 2 kits ( $1 \%$ of all subjects returning kits).

Conclusion A significant number (14\%,125/906) of bowel cancers are detected in those completing 3 kits but this is at the cost of having a screening episode prolonged to almost twice that for subjects obtaining a definitive result after one kit. The intended introduction of faecal immunochemical tests to replace guaiac faecal occult blood tests should allow the use of a simpler and shorter testing algorithm

Disclosure of Interest None Declared. 